\title{
Correlation between TAP detection and common digestive tract precancerous lesions
}

\author{
CHANGQING SUN ${ }^{*}$, FANG DENG ${ }^{*}$, LINGJUN MENG and GUOHUA CHEN \\ Department of Oncology, Dezhou People's Hospital, Dezhou, Shandong 253000, P.R. China
}

Received April 5, 2017; Accepted September 14, 2017

DOI: $10.3892 / \mathrm{ol} .2017 .7496$

\begin{abstract}
The aim of the present study was to investigate the clinical significance of abnormal sugar-chain glycoprotein tumor abnormal protein (TAP) in the screening of common digestive tract pre-cancer colon adenocarcinoma lesions. A total of 50 colitis patients, 50 colon polyp patients and 50 colon adenocarcinoma patients admitted to our hospital from March, 2012 to May, 2014 were included. Fresh blood from patient's fingertips was collected and condensation staining was used to detect TAP expression. Positive expressions of TAP in patients in the colitis, colon polyp and colon adenocarcinoma groups prior to treatment were 6,76 and $92 \%$, respectively. The TAP-positive expression rate comparisons between the three groups were statistically significant $(\mathrm{P}<0.05)$, and TAP-positive expression showed an increasing trend. TAP-positive expression was not significantly correlated with sex, age or ethnic group ( $P>0.05)$. Patient follow-up revealed that the tumor incidence rate in TAP-positive patients was significantly higher than that in TAP-negative in the colitis and colon polyp groups $(\mathrm{P}<0.05)$, and the postoperative tumor recurrence rate in TAP-positive patients was significantly higher than that in TAP-negative in the colon adenocarcinoma group $(\mathrm{P}<0.05)$. TAP had a higher expression in colon pre-adenocarcinoma lesions. Additionally, TAP participated in the processes from intestinal mucosal inflammation to colon polyp formation to tissue canceration, and was correlated with these. Thus, TAP can be used for the screening of digestive tract precancerous lesions.
\end{abstract}

\section{Introduction}

With changes in people's dietary habits and living environment, the incidence of malignant tumors has been on the increase.

Correspondence to: Dr Guohua Chen, Department of Oncology, Dezhou People's Hospital, 1751 Xinhu Street, Dezhou, Shandong 253000, P.R. China

E-mail: phoqxlj9438@163.com

${ }^{*}$ Contributed equally

Key words: colitis, colon polyp, colon adenocarcinoma, abnormal sugar-chain glycoprotein, diagnosis
Surgery, chemoradiotherapy and other therapies are currently used to treat malignant tumors, but through these treatments, patient quality of life decreases, and prognosis remains poor $(1,2)$. Since the pathological features of early stage digestive tract malignant tumors are not obvious, diagnosis can be challenging, and frequently a diagnosis is only made once the tumor has reached middle or late stage, and after the opportunity for curative surgery is no longer available, resulting in poor 5-year survival rates (3). A precancerous lesion is an intermediate link between normal tissue and canceration and, although it is not a malignant tumor, it may have the potential to progress to malignancy (4). Colitis and colon polyps are common precancerous lesions associated with colon adenocarcinoma. Early identification and diagnosis of precancerous lesions is presently a research hotspot, and is likely to play a key role in the treatment of digestive tract malignant tumors in the future.

Abnormal sugar-chain glycoprotein is also known as tumor abnormal protein (TAP), and TAP expression can indirectly reflect the extent and degree of cell canceration $(5,6)$. There have been data suggesting that a relatively high expression of TAP exists in a variety of tumors, such as stomach, colorectal, thyroid and bladder cancers (7-11). However, few reports are available regarding the clinical significance of TAP in the screening of colon pre-adenocarcinoma lesions. In the present study, TAP expression was investigated in patients with colitis, colon polyps, and colon adenocarcinoma to clarify the significance of TAP in common digestive tract precancerous lesions.

\section{Materials and methods}

Study population. A total of 50 colitis patients admitted to the Dezhou People's Hospital (Shandong, China) from March, 2012 to May, 2014 were selected as the colitis group, which comprised 27 males and 23 females, aged 42-65 years (mean, $57.2 \pm 2.6$ ). The colon polyp group included 50 patients with, of whom 26 were males and 24 females, aged 41-66 years (mean 57.6 \pm 2.8 ). The colon adenocarcinoma group comprised 50 patients, of whom 28 were males and 22 females, aged 42-64 years $(57.3 \pm 2.1)$.

Inclusion criteria for the study were: i) Patients who were definitely diagnosed by gastroscope, colonoscopy and pathological examination, ii) patients who agreed to follow-up for dynamic TAP monitoring for 2 years, and iii) who had signed 


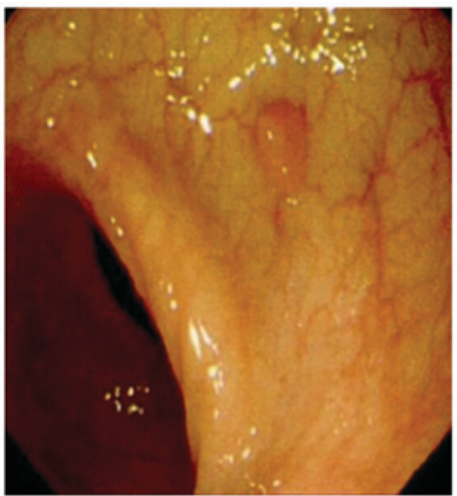

Figure 1. Colon polyp endoscope.

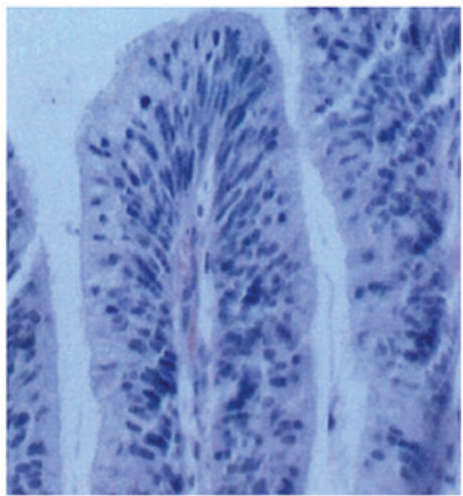

Figure 2. Colon polyp pathology (H\&E; magnification, x400). H\&E, hematoxylin and eosin.

informed consent and agreed to participate in this study. Exclusion criteria for the study were: i) Patients whose digestive tract tumors had transferred and (or) invaded organs outside the digestive tract when they were selected or before surgery, ii) who had other concurrent tumors, iii) patients who had autoimmune disease, iv) with tumors that approached or had reached late stage, and v) with organ incompetence who could not tolerate endoscopy examination. Study approval was obtained from the Medical Ethics Committee of Dezhou People's Hospital.

TAP detection methods. Whole blood from the first and second drops of blood from patient's fingertips was collected, prepared on glass slides, and after being dried naturally, used for detection of TAP. TAP detection reagent (Biosharp Biotech, Hefei, China) was added onto the detection specimen and the condensation staining reaction was performed, followed by microscopic examination to observe results after the reaction was complete and the stain was dried.

Determination of TAP detection results: When TAP exists in blood, it reacts with the reagent and produces a crystal-like condensation product. For TAP-positive, the condensation particle area was $\geq 225 \mu \mathrm{m}^{2}$; for the TAP weakly positive or critical type, the condensation product area was between 121 and $225 \mu \mathrm{m}^{2}$; and for TAP-negative, the crystal-like condensation product was not observed.

$H \&$ E staining of pathological specimens. Pathological tissue specimens of patients in the 3 groups were obtained from

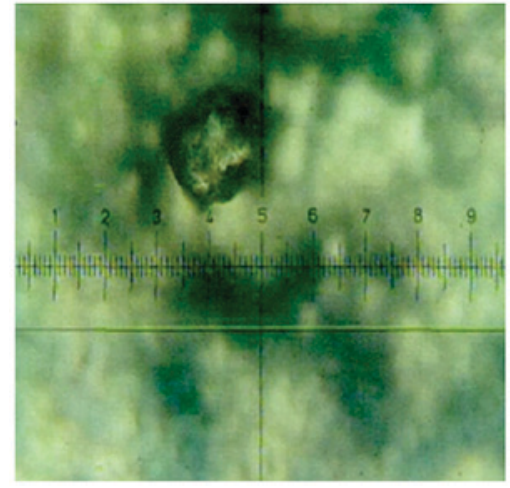

Figure 3. TAP-positive colon polyp (magnification, x400). TAP, tumor abnormal protein.

specimens of large volume excised by surgery or diseased mucosal tissues obtained by gastrointestinal endoscopy biopsy. Pathological sections were made and diagnosed with the assistance of pathology doctors of high seniority.

After pathological sections were frozen and pasted onto slides, the fixative of $95 \% 95 \mathrm{ml}$ alcohol mixed with $5 \mathrm{ml}$ glacial acetic acid was used to fix them for $1 \mathrm{~min}$, and then hematoxylin was used for staining. To determine the results, the cell nucleus was stained blue and cytoplasm was stained pink by hematoxylin.

TAP follow-up monitoring. TAP expression levels were measured at hospitalization and at 15 days after surgery; for patients who were TAP-negative 1-2 years after treatment, detection was performed once every 3 months; for TAP-negative 3-4 years after treatment, detection was performed once or twice a year; for TAP-negative 5 years after treatment, detection was performed once a year; for suspected cases of tumor recurrence and metastasis, or changes in clinical treatment regimen, TAP detection was performed at that time. This study presents data up to 2 years of follow-up.

Statistical analysis. Statistical software SPSS 20.0 (IBM, New York, NY, USA) was used for analysis. Variance analysis was used for comparisons between the 3 groups and a t-test was used for comparison of pairs. Numerical data were presented as percentage $(\%)$, and the Chi-square $\left(\chi^{2}\right)$ test or exact probability test was used for comparison in the 3 groups. A linear trend $\chi^{2}$ test was used to reflect the specimen-positive expression trend. $\mathrm{P}<0.05$ was considered to indicate a statistically significant diference.

\section{Results}

Contrast figures of colon polyp endoscope, pathology and TAP-positive. Results of contrast figures of colon polyp endoscope, pathology and TAP-positive are shown in Figs. 1-3.

TAP-positive expression in colitis, colon polyp and colon adenocarcinoma patients before treatment. TAP-positive expression in colitis, colon polyp and colon adenocarcinoma patients prior to treatment was 6,76 and $92 \%$, respectively, and the positive expression rate comparison between the three groups was statistically significant $(\mathrm{P}<0.05)$ (Table I). 
Table I. TAP-positive expression in colitis, colon polyp and colon adenocarcinoma patients before treatment.

\begin{tabular}{lccccr}
\hline & & \multicolumn{2}{c}{ TAP expression } & \\
\cline { 3 - 5 } Groups & No. & Positive (\%) & Negative (\%) & $\chi^{2}$ & P-value \\
\hline Colitis & 50 & $3(6)$ & $47(94)$ & 7.426 & $<0.05$ \\
Colon polyp & 50 & $38(76)$ & $12(24)$ & & \\
Colon adenocarcinoma & 50 & $46(92)$ & $4(8)$ & \\
Total & 150 & 87 & 63 & \\
\hline
\end{tabular}

TAP, tumor abnormal protein.

Table II. TAP expression trend in colitis, colon polyp and colon adenocarcinoma patients before treatment.

\begin{tabular}{lrccccr}
\hline Groups & No. & Positive cases & Positive (\%) & Score & $\chi^{2}$ & P-value \\
\hline Colitis & 50 & 3 & 6 & 1 & 6.335 & $<0.05$ \\
Colon polyp & 50 & 38 & 76 & 2 & & \\
Colon adenocarcinoma & 50 & 46 & 92 & 3 & & \\
Total & 150 & 87 & & & & \\
\hline
\end{tabular}

TAP, tumor abnormal protein.

Table III. Relationship between TAP expression and clinicopathological parameters of colitis, colon polyp and colon adenocarcinoma.

\begin{tabular}{|c|c|c|c|c|c|c|c|c|c|c|c|c|c|}
\hline \multirow{2}{*}{$\begin{array}{l}\text { Clinicopa- } \\
\text { thological } \\
\text { parameters }\end{array}$} & \multirow[b]{2}{*}{ No. } & \multicolumn{2}{|c|}{ Colitis group } & \multirow[b]{2}{*}{$\chi^{2}$} & \multirow[b]{2}{*}{ P-value } & \multicolumn{2}{|c|}{ Colon polyp group } & \multirow[b]{2}{*}{$\chi^{2}$} & \multirow[b]{2}{*}{ P-value } & \multicolumn{2}{|c|}{$\begin{array}{l}\text { Colon adeno- } \\
\text { carcinoma group }\end{array}$} & \multirow[b]{2}{*}{$\chi^{2}$} & \multirow[b]{2}{*}{ P-value } \\
\hline & & Positive & Negative & & & Positive & Negative & & & Positive & Negative & & \\
\hline \multicolumn{14}{|l|}{ Sex } \\
\hline Male & 81 & 2 & 25 & & & 20 & 6 & & & 25 & 3 & & \\
\hline Female & 69 & 1 & 22 & 0.663 & $>0.05$ & 18 & 6 & 0.402 & $>0.05$ & 21 & 1 & 0.518 & $>0.05$ \\
\hline \multicolumn{14}{|l|}{ Age (years) } \\
\hline$\leq 50$ & 65 & 1 & 23 & & & 19 & 7 & & & 23 & 2 & & \\
\hline$>50$ & 85 & 2 & 24 & 0.007 & $>0.05$ & 18 & 6 & 0.072 & $>0.05$ & 23 & 2 & 0.114 & $>0.05$ \\
\hline \multicolumn{14}{|l|}{ Ethnic group } \\
\hline Ethnic Han & 102 & 2 & 34 & & & 28 & 8 & & & 33 & 3 & & \\
\hline $\begin{array}{l}\text { Other ethnic } \\
\text { groups }\end{array}$ & 48 & 1 & 13 & 0.116 & $>0.05$ & 10 & 4 & 0.105 & $>0.05$ & 13 & 1 & 0.158 & $>0.05$ \\
\hline
\end{tabular}

TAP, tumor abnormal protein.

TAP-positive expression in colitis, colon polyp and colon adenocarcinoma patients before treatment showed an increasing trend (Table II). This suggested that TAP participated in the process from intestinal mucosal inflammation to colon polyp formation to tissue canceration, and it was correlated with them.

Relationship between TAP expression and clinicopathological parameters of colitis, colon polyp and colon adenocarcinoma. TAP-positive expression in all three groups had no significant correlation with sex, age or ethnic group $(\mathrm{P}>0.05)$ (Table III).

TAP follow-up monitoring. TAP follow-up monitoring indicated that, the three TAP-positive patients screened out from the colitis group were examined by gastroscope or colonoscopy, CT, and B ultrasound before treatment, and tumors were not detected. After 2 years of follow-up, one 
Table IV. Comparison of tumor incidence or recurrence between TAP-positive and TAP-negative patients n (\%).

\begin{tabular}{|c|c|c|c|c|c|c|c|}
\hline \multirow[b]{2}{*}{ Groups } & \multirow[b]{2}{*}{ No. } & \multicolumn{2}{|c|}{ TAP-positive } & \multicolumn{2}{|c|}{ TAP-negative } & \multirow[b]{2}{*}{$\chi^{2}$} & \multirow[b]{2}{*}{ P-value } \\
\hline & & Case & $\begin{array}{l}\text { Tumor incidence } \\
\text { or recurrence }\end{array}$ & Case & $\begin{array}{l}\text { Tumor incidence } \\
\text { or recurrence }\end{array}$ & & \\
\hline Colitis & 50 & 3 & $1(33.3)^{\mathrm{a}}$ & 47 & $0(0)$ & 9.832 & $<0.05$ \\
\hline Colon polyp & 50 & 38 & $14(36.8)^{\mathrm{a}}$ & 12 & $1(8.3)$ & 10.561 & $<0.05$ \\
\hline $\begin{array}{l}\text { Colon } \\
\text { adenocarcinoma }\end{array}$ & 50 & 20 & $9(45)^{\mathrm{a}}$ & 30 & $3(10)$ & 11.085 & $<0.05$ \\
\hline
\end{tabular}

Tumor incidence rate or recurrence rate comparisons in TAP-negative patients of the same group, ${ }^{\text {a }}<0.05$. TAP, tumor abnormal protein.

case of colon adenocarcinoma was found. No cases of colon adenocarcinoma were detected in the 47 TAP-negative patients in this group.

The 38 TAP-positive patients screened out from the colon polyp group were examined by gastroscope or colonoscopy, $\mathrm{CT}$, and B ultrasound before treatment, and no tumors were detected. After the 2-year follow-up, 15 cases of colon adenocarcinoma were identified. Only one case of colon adenocarcinoma was detected among the 12 TAP-negative patients in this group. Therefore, the tumor incidence rate in TAP-positive patients was significantly higher than in TAP-negative patients $(\mathrm{P}<0.05)$ (Table IV).

TAP expression was measured in patients in the colon adenocarcinoma group after surgery, of which there were 20 TAP-positive cases, and after 2 years, there were 9 cases of tumor recurrence and metastasis. However, after 2 years, there were only 3 cases of tumor recurrence and metastasis among the 30 TAP-negative patients after surgery. Therefore, the tumor recurrence rate after surgery was significantly higher in TAP positive patients in the colon adenocarcinoma than in TAP negative $(\mathrm{P}<0.05)$ (Table IV).

\section{Discussion}

As common digestive tract cancers are difficult to detect in the early stages, they cause great harm to health. Since the number of cancer cells is relatively small in malignant digestive tract tumors of subclinical stage and nutrient vessels are not yet present, early intervention at this stage can reverse the condition, and 5-year survival rate can surpass $80 \%(12,13)$. Once malignant digestive tract tumors reach middle and late stages, there are no effective treatments, and 5-year survival rate is less than $20 \%(14,15)$. Effective early diagnosis and treatment for malignant digestive tract tumors is currently a major challenge. Research has shown that serum tumor markers, such as CEA, CA19-9 and CA125, are closely associated with tumor occurrence and development. However, the detection of a single tumor marker, such as CEA, CA19-9 or CA125 or traditional tumor marker detection for early diagnosis has poor sensitivity and specificity. For this reason, the above mentioned tumor markers have limited clinical application for malignant tumor screening. The fundamental method to prevent malignant tumors depends on early detection and diagnosis. In previous years, a breakthrough in early diagnosis for malignant digestive tract tumors has been elusive $(16,17)$.

TAP is a type of tumor marker featuring an abnormal sugar-chain (18). When the TAP quantity released by malignant cells reaches a certain level, TAP can be detected in peripheral blood. When TAP shows a positive expression, specific condensation products in peripheral blood can be found, which are different from sundries generated by normal blood $(19,20)$. Thus, we can conveniently determine whether TAP exists in a subject's blood or not and can also evaluate the TAP expression level. As a marker for malignant digestive tract tumors, TAP has the following advantages (21-23): i) As most glycoprotein is distributed on the cell surface and extracellular matrix, when cells become cancerous, the change of sugar chain is detectable; ii) because the $\mathrm{O}$ - and $\mathrm{N}$-sugar chain can be specifically recognized by agglutinin, condensation and sediment phenomena can occur, and the specific shape of condensation products can be observed though a microscope; iii) TAP detection can sensitively identify abnormal sugar chains associated with malignant digestive tract tumors, thus many tumor-related signals can be gathered; and iv) TAP can be detected in malignant tumors of subclinical stage. Previous studies have demonstrated that, TAP expression can be detected up to 2 years earlier than clinical signs, symptoms and malignant lumps (24).

Our investigations have shown that TAP-positive expression rates in patients with colitis, colon polyps and colon adenocarcinoma were 6,76 and $92 \%$, respectively, and that a comparison of the positive expression rate between groups was statistically significant, with TAP-positive expression showing an increasing trend. The TAP-positive expression was not significantly correlated with sex, age or ethnicity in any of the three groups. This result suggests that TAP can effectively distinguish colon adenocarcinoma patients from the large number of patients with colitis and colon polyps. TAP seems to have good specificity for malignant digestive tract tumors, and it may be useful for the early or supplementary diagnosis of this type of malignant tumor.

TAP-negative expression indicates malignant tumors may have been eliminated completely. However, after TAP-negative expression, if TAP weak-positive expression occurs, followed by TAP-positive expression in a short period of time, for example, approximately 1 month, this indicates malignant tumors remain in the body or have started being 
transferred. If TAP-negative expression occurs, later followed by TAP-positive expression after approximately 1 year, recurrence or metastasis of malignant tumors can be determined $(25,26)$. TAP follow-up monitoring showed that the tumor incidence rates in TAP-positive patients in the colitis colon polyp groups were significantly higher than those in the TAP-negative patients. After surgery, the tumor recurrence rate in TAP-positive patients in colon adenocarcinoma was significantly higher than that in TAP-negative patients. This shows that TAP expression is an important index with a high sensitivity to evaluate postoperative recurrence and metastasis of digestive tract tumors belonging to the colon adenocarcinoma type, and through TAP detection, prognosis can be evaluated.

In conclusion, TAP expression is relatively high in colon pre-adenocarcinoma lesions, and TAP actively participates in the process from intestinal mucosal inflammation to colon polyp development to tissue canceration, and is correlated with them. TAP expression can be used for screening of this type of digestive tract precancerous lesion, and has important clinical significance.

\section{References}

1. Zhang H, Hou Y, Xu L, Zeng Z, Wen S, Du YE, Sun K, Yin J, Lang L, Tang $X$ and Liu M: Cytoplasmic drosha is aberrant in precancerous lesions of gastric carcinoma and its loss predicts worse outcome for gastric cancer patients. Dig Dis Sci 61: 1080-1090, 2016.

2. Abangah G, Rahmani A, Hafezi-Ahmadi MR, Emami T, Asadollahi K, Jaafari-Haidarlo A and Moradkhani A: Precancerous histopathologic lesions of upper gastrointestinal tract among dyspeptic patients upon endoscopic evaluations. J Gastrointest Cancer 47: 1-7, 2016.

3. Harmsen S, Meijerman I, Febus CL, Maas-Bakker RF, Beijnen JH and Schellens JHM: PXR-mediated induction of P-glycoprotein by anticancer drugs in a human colon adenocarcinoma-derived cell line. Cancer Chemother Pharmacol 66: 765-771, 2010.

4. Bukowska D: Expression and distribution of zona pellucida proteins 3 and 4 in morphologically abnormal canine oocytes: A confocal microscopic observation-based study. Med Weter 72 68-74, 2016.

5. Albino-Sanchez ME, Vazquez-Hernandez J, Ocadiz-Delgado R, Serafin-Higuera N, León-Galicia I, Garcia-Villa E, Hernandez-Pando R and Gariglio P: Decreased RAR $\beta$ expression induces abundant inflammation and cervical precancerous lesions. Exp Cell Res 346: 40-52, 2016.

6. Go H, Hwang HJ and Nam TJ: A glycoprotein from Laminaria japonica induces apoptosis in HT-29 colon cancer cells. Toxicol In Vitro 24: 1546-1553, 2010.

7. Carta F, Sionis S, Cocco D, Gerosa C, Ferreli C and Puxeddu R: Enhanced contact endoscopy for the assessment of the neoangiogenetic changes in precancerous and cancerous lesions of the oral cavity and oropharynx. Eur Arch Otorhinolaryngol 273: 1895-1903, 2016.

8. Liu Z, Cai J, Yu Y, Fang H, Si Y, Jankee JJ and Shen M: Tumor abnormal protein as a novel biomarker in papillary thyroid carcinoma. Clin Lab 63: 479-485, 2017.

9. Lan F, Zhu M, Qi Q, Zhang Y and Liu Y: Prognostic value of serum tumor abnormal protein in gastric cancer patients. Mol Clin Oncol 5: 216-220, 2016.
10. Zhang L, Guo X, Min Y and Xu J: Tumor abnormal protein (TAP) examination contributes to primary diagnosis of bladder cancer. Int J Clin Exp Med 8: 18528-18532, 2015.

11. Wu XY and Huang XE: Clinical application of serum tumor abnormal protein (TAP) in colorectal cancer patients. Asian Pac J Cancer Prev 16: 3425-3428, 2015.

12. Jin M, Roth R, Rock JB, Washington MK, Lehman A and Frankel WL: The impact of tumor deposits on colonic adenocarcinoma AJCC TNM staging and outcome. Am J Surg Pathol 39: 109-115, 2015.

13. Mroz A and Kiedrowski M: An unusual case of colonic adenocarcinoma development in the region of disseminating lobular breast carcinoma infiltration: Diagnostic approach and review of the literature. Int J Clin Exp Pathol 8: 7470-7474, 2015.

14. Jafferbhoy S, Paterson H and Fineron P: Synchronous gist, colon and breast adenocarcinoma with double colonic polyp metastases. Int J Surg Case Rep 5: 523-526, 2014.

15. Grozdanov P, Hadjidekova S, Dimova I, Nikolova I, Toncheva D, Ganchev G, Zlatkov V and Galabov AS: Characterization of genomic changes in the cervical pre-cancerous lesions and tumors induced by different types of human papillomaviruses. Virusdisease 27: 271-276, 2016.

16. Musiienko AM, Alzahrani S, Simpson JA, Heriot A and Warrier S: Colonic adenocarcinoma encasing the femoral nerve: Complete surgical excision with preservation of function. ANZ J Surg 60: 539-543, 2016.

17. Tatarian T, Arkonac D, Phillips B, Isenberg GA and Goldstein SD: Colonic adenocarcinoma presenting as sigmoidorectal intussusception: A consideration for intussusception reduction. Am Surg 82: 181-182, 2016.

18. Chen J, Ding Z, Peng Y, Pan F, Li J, Zou L, Zhang Y and Liang H: HIF-1 $\alpha$ inhibition reverses multidrug resistance in colon cancer cells via downregulation of MDR1/P-glycoprotein. PLoS One 9: e98882, 2014

19. Jass JR: Colorectal cancer: A multipathway disease. Crit Rev Oncog 12: 273-287, 2006.

20. Herrera-Covarrubias D, Tecamachaltzi-Silvaran MB, Barradas-Moctezuma M, Rosales-Raya JB, Manzo J, García LI, Aranda-Abreu GE, Ismail N, Coria-Avila GA and Hernández ME: Effect of copulation on potentially precancerous prostate lesions, serum testosterone and prolactin levels in rats. Exp Oncol 38: 73-79, 2016.

21. Kamalapuram SK, Kanwar RK and Kanwar JR: Nanotheranostic based iron oxide $\left(\mathrm{Fe}_{3} \mathrm{O}_{4}\right)$ saturated lactoferrin nanocapsules for colonic adenocarcinoma. J Biomed Nanotechnol 12: 1758-1773, 2016.

22. Sun L, Xu S, Liang L, Zhao L and Zhang L : Analysis of ROC: The value of HPV16 E6 protein in the diagnosis of early stage cervical carcinoma and precancerous lesions. Oncol Lett 12: 1769-1772, 2016

23. Halkias C, Sloane J, Ben-Gashir M and Bashir G: Synchronous metastatic omental melanoma and colonic adenocarcinoma: A case report. BMC Res Notes 8: 125, 2015.

24. Jia AI, Lv Y, Guo X, Ren LI and Qin J: Ectopic expression of p33(ING1b) suppresses proliferation and induces apoptosis in colonic adenocarcinoma cells. Oncol Lett 10: 1517-1522, 2015.

25. Almadi MA, Alharbi O, Azzam N, Wadera J, Sadaf N and Aljebreen AM: Prevalence and characteristics of colonic polyps and adenomas in 2654 colonoscopies in Saudi Arabia. Saudi J Gastroenterol 20: 154-161, 2014.

26. Mersakova S, Nachajova M, Szepe P, Kasajova PS and Halasova E: DNA methylation and detection of cervical cancer and precancerous lesions using molecular methods. Tumour Biol 37: 23-27, 2016.

This work is licensed under a Creative Commons Attribution-NonCommercial-NoDerivatives 4.0 International (CC BY-NC-ND 4.0) License. 